\title{
Effect of naloxone on aluminum-induced learning and memory impairment in rats
}

\author{
Sun Shi-lei, MA Guang-yu*, LI Hua Bachelor, Zhu Yun Bachelor, Dong Hong-mei**, XU Xiao-hu** \\ Department of Gerontology, First Affiliated Hospital, Zhengzhou University, ZhengZhou - 450052, *Guangdong Provincial Mental Health \\ Institute, Guangzhou - 510180, **Department of Forensic Medical, Medical College, Shantou University, Shantou - 515031, China
}

Background: Uptake of aluminum may disturb the learning and memory of humans or animals. Naloxone (NAL) has been shown to exert beneficial effects on memory deficits. Aims: We investigated the effects of naloxone on aluminuminduced learning and memory impairment in rats. Settings and Design: Aluminum-induced learning and memory impairment model was established by gavage of Aluminum chloride $(600 \mathrm{mg} / \mathrm{kg})$ for 3 months. Rats were divided into three groups viz. naloxone-treated rats (NAL $0.8 \mathrm{mg} / \mathrm{kg}$, i.p. daily for 7 days), non-treated model rats and normal controls. Materials and Methods: The Morris water maze test was performed to study spatial learning and memory. Longterm potentiation (LTP) of the Schaffer collateral-CA1 synapse was recorded. Aluminum and zinc contents in the hippocampus were assayed with atomic absorption spectrophotometry. Statistical Analysis: Parameters of the hidden and visible platform trials and data of LTP were analyzed using two-way repeated measures ANOVA. Results: In the hidden platform trials, escape latencies of the NAL rats were significantly shorter than that of the non-treated rats ( $P=0.000,95 \%$ confidential interval low bound 14.31 , upper bound 22.68). In probe trails, the number of entries in the target area of the NAL rats $(6.75 \pm 1.28 \mathrm{times} / \mathrm{min})$ was more than that of non-treated model rats $(4.56 \pm 2.16$ times $/ \mathrm{min}$, $P=0.004,95 \%$ confidence interval low bound -3.65 , upper bound -0.788). The magnitudes of LTP recorded in the CA1 pyramidal neurons of the NAL-treated rats were significantly augmented when compared to the non-treated model rats $(P=0.005,95 \%$ confidence interval low bound 0.16 , upper bound 0.84 ). Conclusions: NAL could facilitate spatial learning and memory and enhance LTP in the CA1 region of the hippocampus in aluminum-induced learning and memory impairment in rats.

Key Words: Naloxone, Aluminum, hippocampus, learning and memory, long-term potentiation, Morris water maze

\section{Introduction}

More and more evidence has implicated aluminum (Al) as a neurotoxin, which is involved in the etiology of many neurodegenerative disorders, such as Alzheimer's disease (AD) and dialysis dementia. ${ }^{[1]} \mathrm{AD}$ manifests with insidious and progressive learning and memory loss. It has been reported that the endogenous opioid system in the central nervous system could significantly impair learning and memory, which can be reversed by naloxone (NAL), an opioid receptor antagonist. ${ }^{[2]}$

NAL has been shown to exert beneficial effects on memory deficits in senile dementia and HIV-related dementia patients. ${ }^{[3,4]}$ Our other research showed that NAL could facilitate learning and memory in rats with vascular dementia. ${ }^{[5]}$

We searched for the effects of NAL on long-term potentiation (LTP) of hippocampus and on the behavior of aluminum-induced learning and memory impairment in rats, and aimed to test the hypothesis that NAL can exert beneficial effects on cognitive function.

\section{Materials and Methods}

Animal treatments: All animal treatments were conducted in accordance with the European Communities Council Directive of 24 November 1986 (86/609/EEC). Before the experiment, 27 male and female Sprague-Dawley rats (body weight, 379.1 $20.5 \mathrm{~g}$, certification No. 24101101 conferred by Medical Animal Management Committee, Sichuan Province) were housed for one week for habituation, three animals per cage in a temperature-controlled environment (20$24^{\circ} \mathrm{C}$ ) under a $12: 12 \mathrm{~h}$ light/dark cycle.

Rats were gavaged tap water containing Aluminum chloride (600 $\mathrm{mg} / \mathrm{kg}$ ), into the stomachs daily for 3 months. The containers or tubes were washed with acid. Then rats were randomly divided into two groups viz. NAL-treated model rats and non-treated model rats. Nine rats formed the normal control group. Rats in the NAL group received i.p. injection of NAL $(0.8 \mathrm{mg} / \mathrm{kg})$, daily for 7 days (Days 1- 
7), and the other group received injection of an equal volume of normal saline solution at the same time. From Day 8 the Morris water maze training was carried out.

Morris water maze training The Morris water maze comprised a circular water bath $(2.0 \mathrm{~m}$ in diameter) filled with tap water to a depth of $0.3 \mathrm{~m}\left(22-23^{\circ} \mathrm{C}\right)$ and made opaque. A Perspex platform (11 $\mathrm{cm}$ in diameter) could be placed in any quadrant of the bath. Each trial started by placing the rat gently in the water, facing the edge of the water bath, at the designed location. ${ }^{[5,6]}$

The Morris water maze training comprised four components.

a. Pre-training trials (Days 8-10) — To familiarize the rats with the maze and the escape procedure.

b. Hidden platform trials (Days 11-16) — Each rat was given four consecutive trials per day for 6 days. Starting positions from arbitrarily assigned compass locations were randomized for each rat on each day. The platform was kept in a constant position for all rats. The time taken to reach the platform (escape latency) was noted.

c. Visible platform trials (Days 17-18) - Rats were given four trials per day (inter-trial interval $3-8 \mathrm{~min}$ ) for 2 days with the platform (protruded $1 \mathrm{~cm}$ above the surface of the water) in each of the four quadrants with the same start location and escape latency recorded in each trial.

d. Probe trials - On Day 16, $4 \mathrm{~h}$ after the last hidden platform trial, the platform was removed and the rats were allowed to swim for $1 \mathrm{~min}$ and the number of entries in the target area where the platform used to be was noted. ${ }^{[7]}$

Slice preparations: On Days 19-22, $12 \mathrm{~h}$ after the last water maze training, rats were decapitated one by one at intervals of at least 100 min. The brain was quickly removed. The hippocampus was dissected out and cut into transverse slices $400-\mu \mathrm{m}$ thick. The fresh slices were incubated for at least $90 \mathrm{~min}$ at $33^{\circ} \mathrm{C}$ in artificial cerebrospinal fluid (aCSF $p H$ 7.4) of the following composition (in $\mathrm{mM}$ ): $\mathrm{NaCl}$, $124 ; \mathrm{KCl}, 3.3 ; \mathrm{KH}_{2} \mathrm{PO}_{4}, 1.2 ; \mathrm{MgSO}_{4} .7 \mathrm{H}_{2} \mathrm{O}, 1.5 ; \mathrm{CaCl}_{2}, 2.4 ; \mathrm{NaHCO}_{3}$, 25; D-glucose, 10; bubbled with warm gas $\left(95 \% \mathrm{O}_{2}+5 \% \mathrm{CO}_{2}\right)$.

Electrophysiology: For extracellular recording, a glass electrode was inserted in the pyramidal layer of the $\mathrm{CAl}$ region for the recording of field potential (population spike, PS). A bipolar stimulating electrode was placed in the Schaffer collateral-commissural fiber to activate the LTP. Before the production of tetanus, the population spike evoked by a single pulse was recorded for $15 \mathrm{~min}$ as baseline. Rectangular pulses were delivered at $100 \mathrm{~Hz}$ through the stimulating electrode, as test stimulation, at a strength that gave 70 per cent of the maximal response. ${ }^{[5]}$

Tetanization parameters: LTP was induced by a theta burst stimulation consisting of 3 bursts of 50 pulses ( $100 \mathrm{~Hz}$ frequency, 0.2 msec stimulus duration, 10 msec inter-burst interval). The stimulus intensity was the same as that used for the recordings.

Data analysis: The amplitude of the PS was measured as the distance from the negative peak to the positive. The magnitude of LTP in each preparation was expressed as 20 per cent increase of amplitude of the averaged responses. The data were expressed as a ratio of PS amplitude to baseline.

Determination of $\mathrm{Al}$ and $\mathrm{Zn}$ : The $\mathrm{Al}$ and $\mathrm{Zn}$ content in the hippocampus was determined by atomic absorption spectrophotometry. ${ }^{[8]}$

Statistical analysis: All data were expressed as mean \pm SD. For the hidden and visible platform trials the latencies of each rat on each day were averaged prior to analysis. ${ }^{[6]}$ These parameters and data of LTP were then analyzed using two-way repeated measures ANOVA (RM-ANOVA) with SPSS 10.0 with treatment (NAL) as the inter-subject variable and the time (days or time point after tetanus) as the intra-subject variable. Post hoc comparisons were made using Dunnett's test (all groups compared to control) and Tukey's test (all groups compared with each other).

For the probe trial and the $\mathrm{Al}, \mathrm{Zn}$ content, the data were analyzed using a one-way ANOVA with multiple comparisons as described above.

\section{Results}

$\mathrm{Al}$ and $\mathrm{Zn}$ content in the hippocampus: The $\mathrm{Al}$ content of non-treated model rats $(13.22 \pm 1.47 \mu \mathrm{g} / \mathrm{kg})$ and NAL-treated rats $(10.90 \pm 1.39 \mu \mathrm{g} / \mathrm{kg})$ was elevated as compared to that of normal controls $(4.53 \pm 0.23 \mu \mathrm{g} / \mathrm{kg})\left(\mathrm{F}_{2,24}=58.53, P=0.000\right)$, while there was no significant difference between the former two. There was no significant difference in the $\mathrm{Zn}$ content among the three groups $\left(\mathrm{F}_{2,24}=0.91, P=0.43\right)$.

Hidden platform trials (Days 11-16): Two-way RM-ANOVA showed a significant effect of NAL treatment on the reduction of escape latency $\left(\mathrm{F}_{2,24}=62.303, P=0.000\right)$, (Table 1). Generally, escape latency was significantly longer in the nontreated model rats compared to NAL rats $(P=0.000,95 \%$ confidence interval low bound 14.31, upper bound 22.68, twoway RM-ANOVA followed by Tukey's test) and controls ( $P=0.000,95 \%$ confidence interval low bound 17.65, upper bound 26.01, two-way RM-ANOVA followed by Dunnett's test). On Day 5 and Day 6 trials the escape latency of NAL rats was longer than that of normal controls, while in other trials no significant difference was found $(P=0.636,95 \%$ confidence interval low bound -0.85 , upper bound 7.52, two way RM-ANOVA followed by Dunnett's test).

Visible platform trials (Day 17-18): Two-way RM-ANOVA demonstrated no significant effect of NAL on escape latency in rats in the visible platform trials $\left(\mathrm{F}_{2,24}=1.300, P=0.291\right.$, Table 2).

Table 1: Effect of naloxone on escape latency on the hidden platform trials

\begin{tabular}{lccccc}
\hline $\begin{array}{l}\text { Train } \\
\text { days }\end{array}$ & $\begin{array}{c}\text { Control } \\
\text { group }\end{array}$ & NAL group & Model group & $\mathbf{F}$ & $\boldsymbol{P}$ \\
1 & $29.32 \pm 11.08$ & $40.90 \pm 12.84$ & $89.43 \pm 18.83^{* *_{++}}$ & 38.01 & 0.000 \\
2 & $16.62 \pm 6.44$ & $17.71 \pm 4.95$ & $40.44 \pm 15.87^{*_{++}}$ & 13.65 & 0.000 \\
3 & $7.89 \pm 1.15$ & $7.69 \pm 1.97$ & $19.32 \pm 9.55^{*+}$ & 11.04 & 0.001 \\
4 & $4.59 \pm 0.81$ & $6.77 \pm 2.01$ & $15.29 \pm 4.55^{* *_{++}}$ & 30.18 & 0.000 \\
5 & $4.00 \pm 1.03$ & $7.10 \pm 1.82^{\star}$ & $16.86 \pm 4.48^{* *_{++}}$ & 44.17 & 0.000 \\
6 & $3.21 \pm 0.95$ & $5.54 \pm 2.07^{*}$ & $15.04 \pm 4.89^{* *_{+}}$ & 32.34 & 0.000 \\
\hline
\end{tabular}

NAL stands for naloxone; Number of rats/group $=9$; values are Mean $\pm S D$ in seconds; ${ }^{\star} P<0.05$ vs. control group. ${ }^{* *} P<0.01$ vs. control group. ${ }^{+} P<0.05$ vs. NAL group. ${ }^{++} P<0.01$ vs NAL group.

\begin{tabular}{lccccc}
\hline \multicolumn{5}{c}{ Table 2: Effect of naloxone on escape latency on the visible } \\
platform trials \\
\hline $\begin{array}{l}\text { Train } \\
\text { days }\end{array}$ & $\begin{array}{c}\text { Control } \\
\text { group }\end{array}$ & NAL group & Model group & F & $\boldsymbol{P}$ \\
1 & $8.08 \pm 1.80$ & $11.2 \pm 9.00$ & $7.75 \pm 8.96$ & 0.46 & 0.64 \\
2 & $5.33 \pm 1.93$ & $6.14 \pm 4.25$ & $6.71 \pm 2.66$ & 0.26 & 0.77 \\
\hline
\end{tabular}

NAL stands for naloxone; Number of rats/group=9; values are Mean $\pm S D$ in seconds 
Probe trials: One-way ANOVA showed a significant effect of NAL treatment on the number of entries in the target area in rats $\left(\mathrm{F}_{2,24}=7.991, P=0.002\right)$. The number of entries was significantly increased in the NAL-treated rats $(6.75 \pm 1.28$ times/min) compared to the non-treated rats $(4.56 \pm 2.16$ times/min, $P=0.004,95 \%$ confidence interval low bound -3.65 , upper bound -0.788 , Tukey's test). There wasn't a significant difference in the number of entries between the NAL-treated rats and the normal controls $(7.81 \pm 2.12$ times $/ \mathrm{min}, P=0.636$, $(P=0.000,95 \%$ confidence interval low bound -1.77 , upper bound 1.10, Dunnett's test).

LTP in hippocampal slices of rats: LTP was studied in rats that had been tested in the water maze $(n=9$ slices/group, one slice/rat). After tetanic stimulation, the PS amplitudes increasing more than 20 per cent compared to the mean amplitude before tetanus and lasting more than $30 \mathrm{~min}$ suggested that LTP have been induced in the CA1 region. Also the increased PS evoked by high frequency stimulation in the controls and the NAL-treated rats was seen to be significantly elevated as compared with that of the non-treated model rats ( $P=0.005,95 \%$ confidence interval low bound 0.16 , upper bound 0.84, Tukey's test). No significant difference in PS amplitude was found between the NAL rats and the normal controls $(P=0.328,95 \%$ confidence interval low bound -0.43 , upper bound 0.15 , two way RM-ANOVA followed by Dunnett's test). Two-way RM-ANOVA showed a significant effect of NAL treatment on PS amplitude $\left(\mathrm{F}_{2,24}=7.408, P=0.002\right)$ (Table 3$)$.

\section{Discussion}

Uptake of Al, from Al-treated drinking water over a long period time, may directly enter the brain tissue and disturb the memory and learning of humans or animals. ${ }^{[9]}$ In the present hidden platform trials, the latencies of the non-treated model rats were significantly longer than that of the normal controls, the number of entries in the target area in probe trials was significantly decreased, and the magnitudes of LTP were significantly decreased, which showed that long-term (3

\begin{tabular}{|c|c|c|c|c|c|}
\hline $\begin{array}{l}\text { Time } \\
\text { after } \\
\text { tetanus } \\
\text { (min) }\end{array}$ & $\begin{array}{l}\text { Control } \\
\text { group }\end{array}$ & NAL group & Model group & $\mathbf{F}$ & $P$ \\
\hline 5 & $1.69 \pm 0.33$ & $2.28 \pm 1.42$ & $1.37 \pm 0.30^{\star+}$ & 5.26 & 0.008 \\
\hline 10 & $1.72 \pm$ & & $4^{\star *_{+}}$ & 5.58 & 0.006 \\
\hline 15 & $1.77 \pm 0.37$ & $2.14 \pm 1.24$ & $1.21 \pm 0.27^{\star_{*_{+}}}$ & 6.75 & 0.003 \\
\hline 20 & $1.80 \pm 0.40$ & $2.13 \pm 1.29$ & $1.18 \pm 0.30^{* *+}$ & 6.62 & 0.003 \\
\hline 30 & $1.79 \pm 0.37$ & $1.98 \pm 1.12$ & $1.14 \pm 0.33^{* *_{+}}$ & 6.99 & 0.002 \\
\hline 40 & $1.85 \pm 0.57$ & $1.94 \pm 1.04$ & $1.08 \pm 0.38^{* \star_{++}}$ & 7.74 & 0.001 \\
\hline 50 & $1.88 \pm 0.63$ & $1.84 \pm 0.94$ & $0.93 \pm 0.41^{* *++}$ & 10.73 & 0.000 \\
\hline 60 & $1.89 \pm 0.67$ & $1.78 \pm 0.89$ & $0.86 \pm 0.43^{*_{++}}$ & 12.01 & 0.000 \\
\hline
\end{tabular}

NAL stands for naloxone; LTP stands for Long-term potentiation; Number of slices/group=9(one slice/rat); values (PS amplitude/baseline) are Mean $\pm S D$. ${ }^{*} P<0.05$ vs. control group. ${ }^{*} P<0.01$ vs. control group. ${ }^{+} P<0.05$ vs. NAL group. ${ }^{+}+P<0.05$ vs. NAL group. months) intake of $\mathrm{Al}$ could induce learning and memory deficits in rats.

It was reported that compared to other brain tissue, the hippocampus had the highest concentration of $\mathrm{Al}{ }^{[10]} \mathrm{As}$ an important target organ of $\mathrm{Al}$ neurotoxicity, the hippocampus is a crucial element of the neurobiology basis of higher cognitive function. ${ }^{[11]}$ In the present research, the $\mathrm{Al}$ content in the hippocampus of model rats was elevated as compared with that of normal controls, which shows high accumulation of $\mathrm{Al}$ in the hippocampus.

After treatment with NAL, the latencies of model rats in the hidden platform trials were notably reduced. This was not confounded by other factors that could cause interference, such as non-specific deficits in visual and motor function, because there was no significant difference among the groups in the visible platform trials.

In probe trials, the increase in the number of entries of the NAL-treated model rats suggests the enhancement of memory. Taken together, our results indicate that NAL facilitates aluminum-induced spatial learning and memory deficit in rats.

LTP is supposed to be a well-known cellular model of learning and memory. ${ }^{[12]}$ The factors that impair memory and learning decrease the magnitude of LTP, while the drugs or factors that facilitate LTP improve memory. ${ }^{[13]}$

To determine whether NAL affected synaptic plasticity, LTP was studied in hippocampal slices from the rats. Recording was made in the CA1 section, because LTP occurring in the Schaffer collateral (SC)- CA1 pathway is believed to be important for spatial memory. ${ }^{[14]}$ In the present study, the magnitudes of LTP of model rats were significantly decreased, which confirmed results from other laboratories that Aluminum impairs hippocampal LTP in rats in vitro and in vivo. ${ }^{[15]}$ The PS amplitude of the NAL-treated rats was notably augmented compared to that of non-treated model rats, indicating that NAL could enhance the magnitude of LTP in the CA1 region of rats.

Opioid peptides exist in the hippocampal neurons and modulate hippocampal plasticity. ${ }^{[16]}$ Activation of kappa receptors blocked the induction of LTP, which can be reversed by NAL. ${ }^{[17]}$ Aluminum was found to increase the permeability of the bloodbrain barrier to $\beta$-endorphin which impairs memory and learning. ${ }^{[2,18]}$ It is presumed that NAL may facilitate the Al-induced memory and learning deficit of rats because of its endogenous opioid antagonistic properties.

Apart from beingan opioid receptor antagonist, NAL has other pharmacological effects: (1) NAL exerts neuroprotective effects by decreasing superoxide production, ${ }^{[19]}$ while $\mathrm{Al}$ enhances the production of superoxide radicals which may account in part for the biological toxicity associated with $\mathrm{Al}^{[20]}$ (2) Cholinergic neurotransmission is related closely to learning and memory. NAL elevates the Acetylcholine release in the hippocampus of rat. ${ }^{[21]}$ Aluminum decreases the Choline acetyltransferase content and Acetylcholine activity of mice, 
which may be one of the important mechanisms of its neurotoxity. ${ }^{[22]}$

There was no significant difference in the $\mathrm{Al}$ and $\mathrm{Zn}$ content between the non-treated model rats and the NAL-treated rats, which suggests that the beneficial effects of NAL on cognitive function may not be through decreasing the level of $\mathrm{Al}$ or $\mathrm{Zn}$ in rats.

In conclusion, our findings demonstrate that NAL could facilitate the spatial learning and memory and augment LTP of Schaffer collateral-CA1 synapse in the hippocampal slice preparations of aluminum-induced learning and memory impaired rats.

\section{Acknowledgment}

This work was supported by the Natural Science Foundation of Guangdong Province, China

\section{References}

1. Flaten TP. Aluminium as a risk factor in Alzheimers disease, with emphasis on drinking water. Brain Res Bull 2001;55:187-96.

2. Sollertinskaya TN. Comparative study of the roles of ACTH and $\beta$-endorphin in regulating conditioned reflex activity in the hedgehog. Neurosci Behav Physiol $1997 ; 27: 663-71$.

3. Replydar KS, Kamaraju LS, Dingfelder J, MeMahon J, Gollapudi S, Wilson WH, et al. $\beta$-endorphin enhances the replication of neurotropic human immunodeficiency virus in fetal perivascular microglia. J Neuroimmunol 1995;61:97104.

4. Reisberg B, Ferris SH, Anand R, Mir P, Geibel V, de Leon M.J. Effects of naloxone in senile dementia: A double-blind trial. N Engl J Med 1983;308:721-2.

5. Shilei S, Xiaohu X, Guangyu M, Hong X. Effect of NAL on cognitive function in vascular dementia in rats. Indian J Med Res 2002;115:265-71.

6. Conway EL. Brain lesions and delayed water maze learning deficits after intracerebroventricular spermin. Brain Res 1998;800:10-20.

7. D'Hooge R, Van Dam D, Franck F, Gieselmann V, De Deyn PP. Hyperactivity, neuromotor defects, and impaired learning and memory in a mouse model for metachromatic leuckodystrophy. Brain Res 2001;907:35-43.

8. Kraemer HJ, Breithaupt H. Quantification of desferrioxamine, ferrioxamine and aluminoxamine by post-column derivatization high-performance liquid chromatography. Non-linear calibration resulting from second-order reaction kineties. J Chromatogr B Biomed Sei Appl 1998;710:191-204.

9. Walton J, Tuniz C, Fink D. Uptake of trace amount of aluminum into the brain from drinking water. Neurotoxincology 1995;16:187-90.

10. Fattoretti P, Bertoni-Freddari C, Balietti M, Giorgetti B, Solazzi M, Zatta P Chronic aluminum administration to old rats results in increased levels of brain metal ions and enlarged hippocampal mossy fibers. Ann N Y Acad Sci 2004;1019:44-7.

11. Savage LM, Buzzetti RA, Ramirez DR. The effects of hippocampal lesions on learning, memory, and reward expectancies. Neurobiol Learn Mem 2004;82:109-19.

12. Barria A, Muller D, Derkach V, Griffith LC, Soderling TR. Regulatory phosphorylation of AMPA-type glutamate receptors by CaM-KII during long-term potentiation. Science $1997 ; 276: 2042-5$.

13. Bliss TVP, Collingridge GL. A synaptic model of memory: Long-term potentiation in the hippocampus. Nature 1993;361:31-9.

14. Silva A.J, Paylor R, Wehner JM, Tonegawa S. Impaired spatial learning in alpha calcium-calmodulin kinase II mutant mice. Science 1992;257:206-11.

15. Platt B, Carpenter DO, Busselberg D, Reymann KG. Aluminum impairs hippocampal long-term potentiation in rats in vitro and in vivo. Exp Neurol 1995; $134: 73-86$.

16. Morris B.J, Johnston HM. A role for hippocampal opiodis in long-term functional plasticity. Trends Neurosei 1995;18:350-5.

17. Terman GW, Wanger JJ, Chavkin C. Kappa opioids inhibit induction of longterm potentiation in the dentate gyrus of the guinea pig hippocampus. J Neurosci 1994;14:4740-7.

18. Banks WA, Kastin A.J. Peptide transport systems for opiates across the bloodbrain barrier. Am J Physiol 1990;259:1-10.

19. Chang RC, Rota C, Glover RE. A novel effect of an opioid receptor antagonist, naloxone, on the production of reactive oxygen species by microglia: A study by electron paramagnetic resonance spectroscopy. Brain Res 2000;854:224-9.

20. Adler A, Murray S. The effect of aluminum on the Vanadium-mediated oxidation of NADH. Nephron 1995;69:34-40.

21. Mizuno T, Kimura F. Medial septal injection of NAL elevates acetylcholine release in the hippocampus and induces behavioral seizures in rats. Brain Res 1996; $713: 1-7$

22. Wu Ying Hong, Zhou Zhong Ming, Xiong Yu Lan, Wang Yanli, Reply Jianhua. Effects of aluminum potassium sulfate on learning, memory, and cholinergic system in mice. Acta Pharmacologica Sinica 1998;19:509-12.

Accepted on 10.10.2004. 